\title{
CATE: A Circuit Analysis Tool for Education
}

\section{Dr. Fred W. DePiero, California Polytechnic State University, San Luis Obispo}

Dr. Fred DePiero received his B.S. and M.S. degrees in Electrical Engineering from Michigan State University in 1985 and 1987. He then worked as a Development Associate at Oak Ridge National Laboratory until 1993. Fred began working on his Ph.D. at the University of Tennessee while still at ORNL, and completed it in May 1996. Fred joined the faculty at Cal Poly in September of 1996. He is presently serving as the Associate Dean for Student Success in the College of Engineering.

\section{Mr. K. Clay McKell, California Polytechnic State University, San Luis Obispo}

Clay McKell earned his B.S. degree, summa cum laude, in mechanical engineering from UCLA in 2006. He earned his M.S. degree in mechanical engineering from UCLA in 2007. He is currently pursuing a Ph.D. in electrical engineering from the University of Hawaii at Manoa and teaching as a lecturer in the Electrical Engineering Department at California Polytechnic State University, San Luis Obispo. His research interests include distributed control of multi-agent networks as well as STEM education practices that foster diversity and equity.

\section{Dr. Bridget Benson, California Polytechnic State University, San Luis Obispo}

Bridget Benson received a Bachelor's degree in Computer Engineering at California Polytechnic State University San Luis Obipso in 2005, a Master's degree in Electrical and Computer Engineering at the University of California Santa Barbara in 2007 and a PhD degree in the Computer Science and Engineering at the University of California San Diego in 2010. She is currently an Assistant Professor in the Electrical Engineering Department at California Polytechnic State University San Luis Obipso. Her research interests span engineering education, embedded systems, and ecological monitoring. 


\section{CATE: A Circuit Analysis Tool for Education}

This paper describes a new teaching and learning tool for linear circuit analysis, CATE (the Circuit Analysis Tool for Education). CATE supports both active and passive learning for students. In passive mode, it generates circuits, transparently formulates necessary equations, and solves for unknowns. Students can vary the difficulty of the problem and choose the desired solution method: equivalent circuits, nodal analysis, or mesh analysis. In active mode, CATE generates and grades practice problems in areas including DC and AC analysis, Thevenin modeling, and superposition. CATE is a free web resource that works in a variety of browsers, at YourLearningCoach.com. Student evaluations of the system suggest that it has already become a useful study tool. We proceed to describe the implementation of the CATE software including such novel features as guaranteed "nice" numeric values and platform-independence.

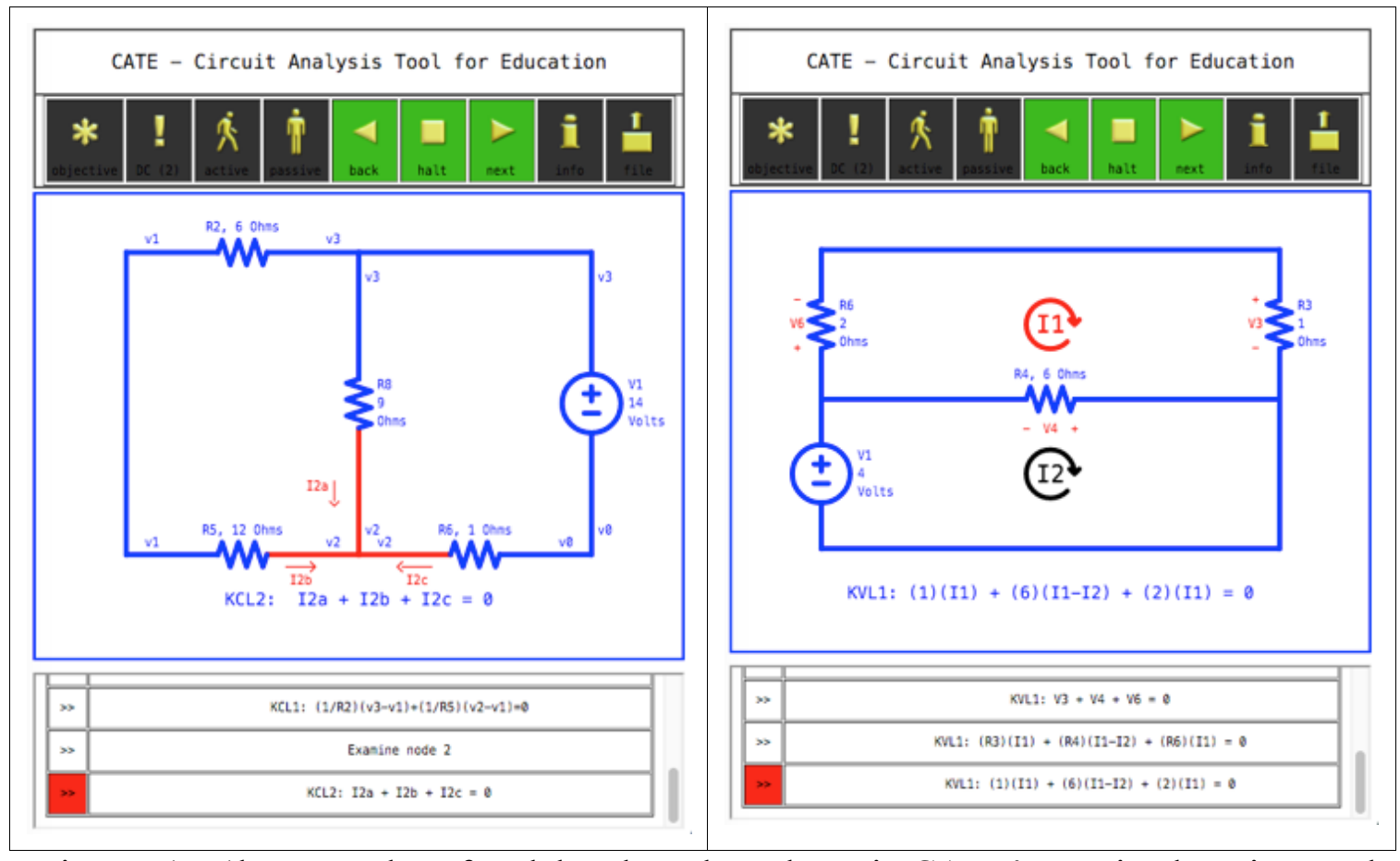

Figures 1a, 1b. Examples of nodal and mesh analyses in CATE's passive learning mode. Students are guided step-by-step through the analysis of generated circuits. Relevant voltages, currents and components are highlighted with each corresponding equation.

The first two sections below discuss goals, features and learning objectives. In Section 3 we include some details of our implementation. Results of student opinion surveys are in Section 4, describing our assessment of design and usage issues. Screenshots of the CATE website appear throughout.

\section{Goals}

Our goals for CATE are multifaceted, and are intended to benefit both teachers and learners. From the student perspective, CATE provides both active and passive learning 
opportunities ${ }^{1}$ Usage is self-directed to provide flexibility and is driven by a student's needs. ${ }^{2,3}$ CATE can help reinforce lecture material and provides many practice problems.

CATE is also designed to provide direct benefits to instructors. It can serve as a standalone learning resource, much as a second textbook would. ${ }^{5}$ It can also provide a platform for in-class examples. Instructors can use CATE to provide in depth, step-bystep details or to provide broad overviews that include many different circuits. It can also be used to create videos for a hybrid course ${ }^{6,28}$ and can help diversify the technology options for a class ${ }^{7}$ at no added cost. As a free web-based tool ${ }^{11}$ CATE can be readily accessed to compliment and extend the resources of a traditional course. ${ }^{3}$
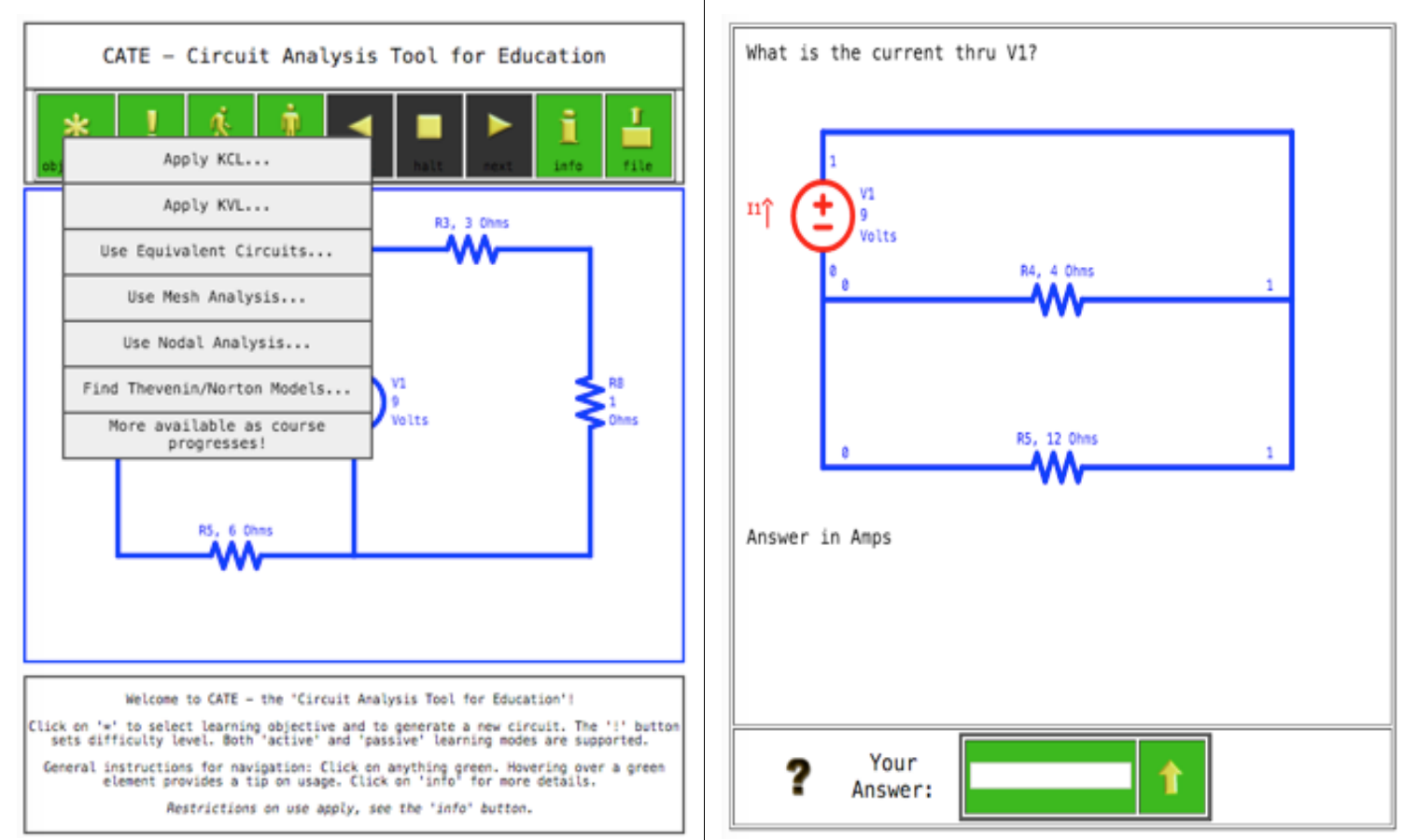

Figures 2, 3. Example circuits are generated based on a selected learning objective (left). Active learning is supported via exercises that are automatically graded (right) and implemented in a low-stakes fashion, without consequence to a student's course grade.

\section{Functionality and Learning Objectives}

CATE provides a large number of example circuits (self generated with random variations) for both DC and AC analysis. The style of circuit varies depending on a student's choice of learning objective (Fig 2) and difficulty level. Styles vary for nodal analysis versus Thevenin equivalents, for example. Schematic templates are used to generate circuits and ensure that an appropriate circuit is generated for a selected learning objective. See the section on implementation for more details.

In passive mode, equations appear in both symbolic (Fig. 1a) and numeric form (Fig. 1b) in a step-by-step automated tutoring. At each step relevant circuit quantities such as mesh 
currents and KVL voltages are highlighted in the schematic. Simplified equations and final numerical results (e.g. mesh current values) are also presented.

\subsection{Learning Objectives}

CATE supports the following learning objectives.

- Identify series and parallel elements; shorts and open connections

- Identify the number of nodes in a circuit and the extent of each

- Identify where KVL and KCL can be applied and formulate equations with these laws

- Analyze circuits using equivalents, relating quantities between versions

- Analyze circuits using mesh current and nodal voltage methods proficiently

- Analyze both AC and DC circuits

- Find Thevenin and Norton circuit models

- Use superposition in circuit analysis

- Use results from an analysis method to compute other electrical quantities

- Predict changes in electrical quantities due to changes in component values

\subsection{Pedagogical Features}

Misconceptions on topics such as identifying a node; identifying series and parallel elements; and handling shorted and open connections are common for beginning students. ${ }^{4}$ CATE addresses these misconceptions in a variety of ways. For example, it identifies series and parallel elements by highlighting relevant nodes and then combining elements to form an equivalent circuit. (See Figures 6.d to 6.p) Shorts are also highlighted and the shorted components are subsequently removed. Open connections are similarly trimmed out of the circuit. These sorts of circuit modifications are presented step-by-step to the student with text explanations and equations, and corresponding circuit diagram highlights. Other examples of the foundational concepts of nodes, and series / parallel configurations include KCL and finding the Thevenin resistance. (Figs 1a, 4b.)

To accommodate students' diverse learning styles CATE incorporates Kolb's 4MAT approach." ${ }^{9,12}$ Kolb's "What," "Why" and "How" stages of the learning process appear explicitly in passive mode. Students can practice the "How" steps of analysis in active learning mode and may explore "What If" by altering component values or source frequency of a given circuit, for example.

Unlike a prerecorded video, CATE supports more individualized paths through the learning process, as driven by a student's need. For example, after a student selects a particular learning objective (e.g. nodal analysis) they can review it step-by-step. However, they can also select alternative methods for that same circuit. Thus students have an opportunity to fall back to prior foundational techniques as needed. 
Similarly, if a student tries using the active mode for a given circuit and then later decides they want to see a detailed solution, they can return to passive mode for a step-by-step presentation. The variable difficulty levels in CATE, stepwise explanations and the ability to iteratively fallback and move forward are scaffolds that support the learner's progress. ${ }^{26}$

\subsection{Nearest Neighbors}

The nearest neighbor to CATE appears to be a system developed by Skromme, et al. ${ }^{16,17,18}$ Both systems generate circuits and the analyses include symbolic and numeric equations, along with final results. As Skromme describes, prior to their system, methods of computer-aided instruction have provided only partial prototypes, and rarely have prior systems included the ability to generate example circuits. ${ }^{19,23}$ CATE shares many attributes of Skromme's system, and includes some additional features. Beyond Skromme's work, CATE includes the method of analysis by equivalent circuits as well as modeling via Thevenin and Norton techniques. Both CATE and Skromme's system generate circuits, but CATE also has an option to produce 'nice' numerical values that allows a user to complete some analysis without using a calculator. CATE also allows users to share circuits via download and upload to promote collaboration. A feature that Skromme supports which we do not is the ability to handle dependent sources. This is planned for a future release of CATE.

To better clarify the functionality and intent of CATE, we compare it to some other common systems. (These comments hold for Skromme's system as well). To begin with, CATE is not SPICE. ${ }^{15}$ CATE solves for the numerical value of electrical quantities, as does SPICE. However it also presents equations in symbolic form (Fig. 1a) and with numeric coefficients (Fig. 1b). This mimics how we teach circuit analysis in lecture whereas SPICE obscures the solution process. ${ }^{24,25}$ Furthermore CATE is not a MOOC. ${ }^{14}$ Usage of CATE is self-directed by the student, as opposed to offering a predetermined sequence of material. Lastly, CATE is not a course management system such as Moodle. ${ }^{13}$ CATE is focused on the pedagogical delivery of specific learning objectives, and includes a virtually unlimited number of internally generated circuits, with solutions and explanations. CATE is not a general-purpose tool to help organize a course.

\section{Implementation}

CATE employs data structures that describe both a physical representation (with locations on a schematic and component types, values and labels) and a logical representation (with electrical nodes and branch connectivity). Special tags are also included, for example to flag if a particular node should be grounded. To verify results, a new circuit is automatically analyzed by both the mesh and nodal techniques. The results from each of these methods must match within a specified tolerance (accounting for possible polarity differences).

Our implementation of mesh and nodal analysis parallels common practice. ${ }^{24,25}$ However our method of analysis by equivalent circuits may be somewhat non-standard. We have 
defined a systematic procedure, in place of what is typically an ad hoc technique. See Figures 6.a through 6.p for an example of the process. CATE simplifies a circuit by combining series and parallel elements. At each stage of the simplification process a new circuit is pushed onto a stack. Simplification continues until only a single mesh with one source and one passive element remain. Then Ohm's law is employed. After this, voltage and current information is propagated back to each more complicated version of the circuit as it is popped off the stack. For example, if an equivalent element was formed by a series combination, then the current in the equivalent must be equal to the current in the combined elements. If an equivalent element was formed by a parallel combination, then the voltages must be equal. The use of this technique is of course limited to circuits that can be reduced by series and parallel equivalents.

CATE makes the process of collapsing (and then expanding) a circuit using series and parallel equivalents transparent to the student in the passive learning mode. This has the advantage of concretely demonstrating correct use of voltage and current dividers, which are notoriously difficult concepts in introductory circuits classes. ${ }^{27}$

CATE is written in Javascript. ${ }^{20}$ We integrated the Math.js ${ }^{21}$ numerical package to solve simultaneous equations with complex values for AC analysis. Because CATE runs in a browser, it places no demand on a server or network, except during the initial page download. This benefits users with limited connectivity. A subsequent release will place slightly higher demands on networks due to longitudinal tracking capabilities that monitor usage for assessment purposes.

\subsection{Circuit Generation}

Our method of generating circuits involves the use of templates. These templates define degrees of freedom used during the circuit generation process. This approach supports a wide variation in circuit structure while still constraining the generated circuit to ensure it is appropriate for a given learning objective (e.g. Thevenin versus mesh analysis, or AC versus DC).

The example template in Figure 4 includes 12 elements of variable types. Two active elements (circular) define the existence of either a voltage or current source. Ten passive elements (rectangles) define resistors, inductors, capacitors or wires. The digits by each element indicate the number of possible component types; the total number of prototype networks in the figure (for this single template) is over 1.7 million. Note the option of a wire was avoided for some elements, to avoid shorting portions of the network. Circuits with more meshes or more elements per branch are possible, as are circuits with shorts or open connections. Allowing for shorts, the topology shown in Figure 4 could yield $4^{11}$, or over 4 million prototype networks, before specific component values are selected. See Figure 5 for examples of circuits generated by CATE, via various templates. 


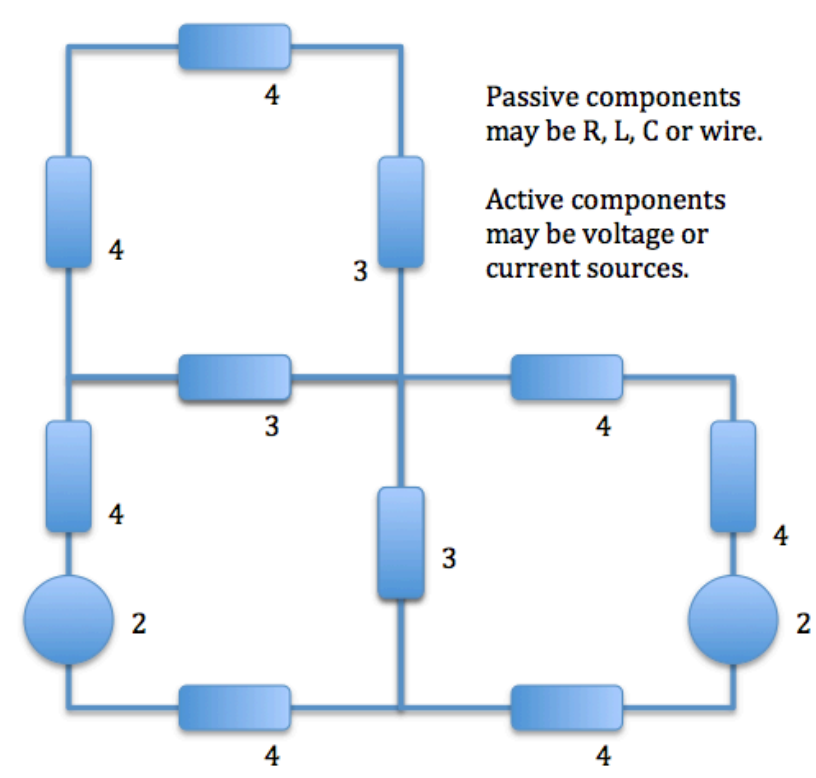

Figure 4. This circuit template has two sources (circles) and 10 passive elements (rectangles). The number of permitted types for each element is shown, resulting in over 1.7 million possible prototype networks (before component values are assigned).

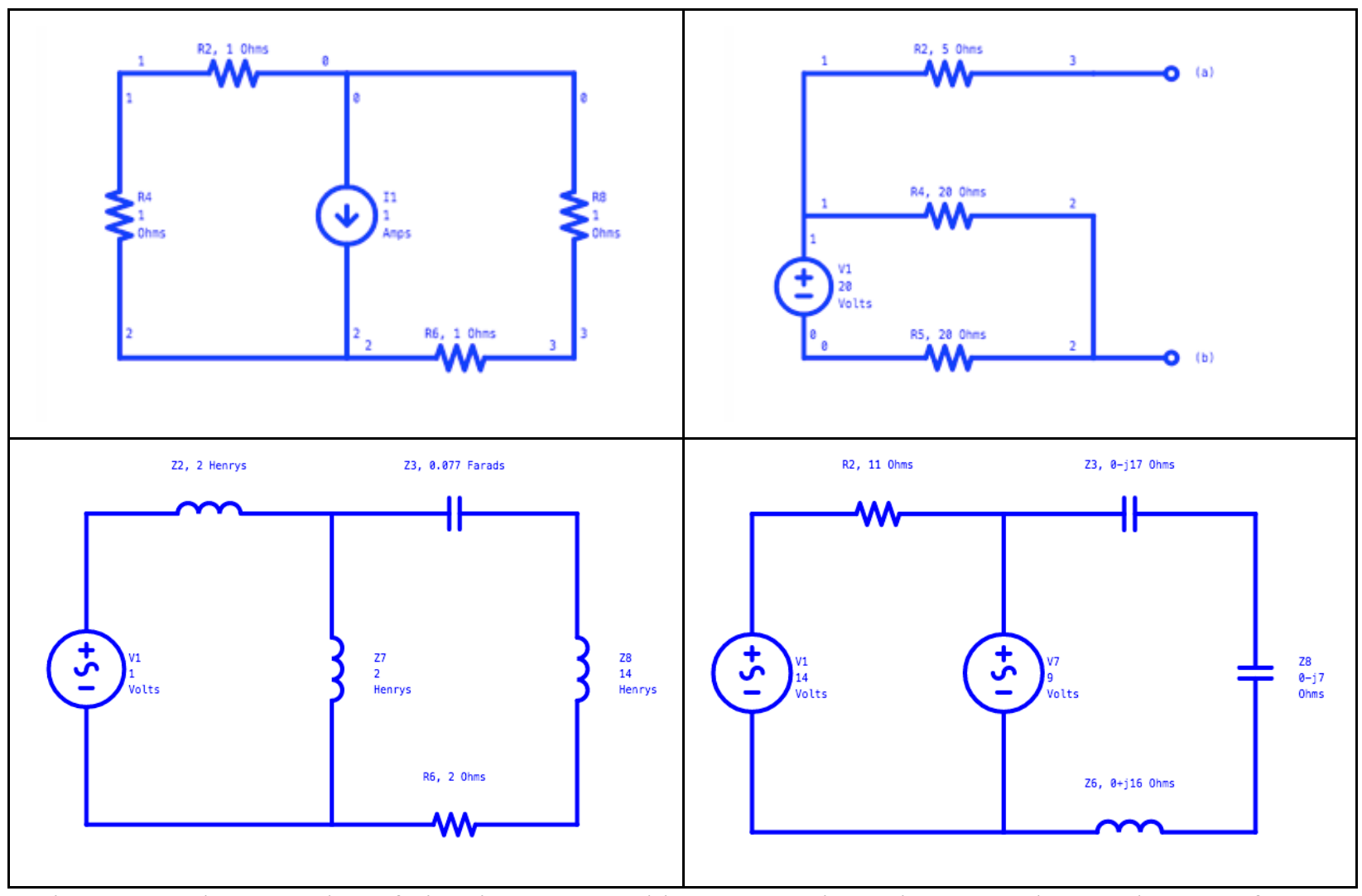

Figure 5a-5d. Examples of circuits generated by CATE via various templates. The use of templates permits a large number of circuits to be generated, while constraining the style of a circuit to be appropriate for a given learning objective. 


\subsection{Generating Circuits with "Nice" Values for Components, Voltages and Currents}

CATE leverages the method of equivalent circuits to generate DC circuits with integer valued component values, voltages and currents. Our technique is based on the logic used to form equivalent circuits by combining series and parallel elements. "Nice" component values are assigned beginning with the circuit in its most simplified equivalent version, for example having a voltage source and single equivalent resistance. CATE randomly picks a source current and total resistance that are integer-valued, resulting in an integervalued source voltage. This and subsequent steps overwrite original component values. Step by step CATE then reverses the process of circuit simplification. As series and parallel reductions are each undone, component values are selected that maintain integer valued components and currents. If components become less than one, then all components are scaled up in value. (For parallel resistors, a separate program was developed that predetermined a number of cases having integer-valued combinations of resistors.) As described this algorithm generates integer values; however in the present implementation we opted for a variation that yields integer currents in milliamps. This approach yields circuits with more typical component values. In the present implementation we also allow for rational values of current.

\section{Assessment}

The goal of our initial assessment was to examine students' opinion of the usefulness of CATE to their learning process. We were also curious about students' perception of various features. With regard to the learning process, we wanted to investigate:

- Interest in using CATE for a low stakes versus high stakes environment

- Relative interest in active versus passive learning modes

- Value of including Kolb's 4MAT

- Relative interest in CATE compared to other learning resources

- Possible issues or concerns with our implementation

\subsection{Assessment Survey}

Prior to taking the survey, students had the option to watch a five-minute introductory video and also invited them to explore the CATE environment directly. ${ }^{10,11}$ Based on students' general comments in the survey, many respondents had already tried CATE in our EE 201 basic circuits course. Note this is a service course for non-majors. Results below are from 59 respondents.

For the following survey questions on CATE features, students rated items with this Likert scale:

- (5) Strongly agree

- (4) Agree

- (3) Neutral

- (2) Disagree

- (1) Strongly disagree 
Students were asked to rank CATE's features. Questions are sorted based on students' weighted average response

- (4.5) Passive learning mode, where CATE shows you step-by-step analysis and final answers

- (4.4) The ability to generate a large number of practice problems

- (4.3) Step-by-step explanations that include 'what, why, how'

- (4.3) Circuits with 'nice' voltage, current and component values (as opposed to values that are arbitrary fractions)

- (4.3) Full compatibility with iPad, tablets and similar devices (planned)

- (4.2) Active learning mode, where you work through a problem and then CATE checks your answer - with no impact on your course grade

- (4.1) Videos that use CATE to explain key concepts (e.g. mesh or nodal analysis)

- (4.1) The ability to edit schematics and build your own circuits (under consideration for a future release)

- (4.0) The ability to modify component values of a CATE circuit, and see the impact of these changes

- (4.0) Integrating CATE with an FAQ discussion board (under consideration)

- (3.7) Homework or quizzes that use CATE, that would impact your course grade

- (3.3) Download and sharing .cate files, as a means to share circuit problems with your study group

- (3.3) The ability to see your usage and performance relative to your classmates (under consideration for a future release)

Students used the following Likert scale for the remaining survey questions:

- (5) Yes, definitely

- (4) Yes, probably

- (3) Neutral / Not applicable

- (2) No, probably not

- (1) No, definitely not

Student ratings of the perceived benefit of CATE when learning basic circuits

- (4.5) Provide a more efficient way to learn basic circuit analysis

- (4.4) Promote individual study

- (4.0) Encourage spending more time studying

- (3.6) Promote group study

Student ratings of how they would prefer using CATE

- (4.7) Review and practice methods before an exam?

- (4.3) Reinforce learning after a topic is presented in class?

- (4.0) Review methods when they are needed in a future course?

- (2.8) Learn a new circuit analysis technique before it is presented in class? 
Student rankings of how would you prefer to reinforce their understanding after first learning a topic in lecture

- (4.4) Review your class notes

- (4.3) Use CATE

- (3.7) Google search

- (3.7) Review lecture capture videos

- (2.8) Read Schaum's Outline (or similar source)

- (2.4) Read the textbook

\subsection{Evaluation of Survey Results}

Students responded favorably on the questions concerning basic features. Best ranked included the passive learning mode (4.5) and ability to generate a large number of practice problems (4.4). Active learning was rated fairly high as well (4.2) although lower than passive. Students appear to prefer using CATE's practice problems as opposed to creating their own - we continue to examine this. They ranked the interest in full compatibility with mobile devices relatively high as well (4.3). One of the lowest ranked features (3.7) was using CATE for homework or quizzes that have grading consequences in their course. They also reported little interest in comparing their own usage and performance with CATE relative to their peers. This result was counter-intuitive given a typically competitive spirit among the students!

Concerning the perceived benefit of CATE, students rated the use for individual study (4.4) as a more attractive option than for group work (3.6). This is consistent with prior studies. ${ }^{3}$ Students also thought that CATE would improve their efficiency when learning basic circuits (4.5). With regard to when they might employ CATE in the learning process, students ranked preparing for an exam the highest (4.7). They preferred using CATE to reinforce their learning after an initial exposure in class (4.3), versus learning it first with CATE (2.8).

Relative to other learning resources, CATE fared quite well (4.3), second only to students reviewing their own notes (4.4). These options were above a Google search (3.7), reviewing lecture capture videos (3.7), using Schaum's Outline ${ }^{5}(2.8)$ or the class textbook (2.4). The relative ranking of CATE above the required textbook indicates that the modern college student is hungry for a high-quality, low-cost alternative to traditional learning resources. We are pleased with these assessment results, as it appears that students feel CATE is beneficial to their learning, and that it is a resource they might use. Students also indicated that the user interface should be redesign for easier use (4.11). This is an active follow up to close the loop with our initial assessment process. Other recommendations by students included more introductory videos and possibly a pre-test to guide student usage, although this was getting into the neutral category (3.6). 


\section{Conclusions and Future Plans}

The current version of CATE provides a low stakes learning environment. Assessment by students of our system design and has been positive overall. Potential uses by students include reviewing concepts from lecture and preparing for exams. In a future update we plan to establish user management features. This will permit longitudinal tracking of individual users and allow statistics on usage and performance for a group of students to be computed. We are also considering a pre-test to help guide students when using CATE.

We hypothesize that CATE will help foster student learning. Furthermore we anticipate that some methods of using CATE will be more beneficial than others. We expect the following sequence may be near optimal.

1. Student is introduced to concepts in lecture

2. Student uses passive mode to ensure a foundational understanding of concepts

3. Student switches to active mode and progressively increases difficulty levels

4. Student switches to passive mode to review examples when preparing for an exam

Our initial assessment results indicate that students may be following the above scenario on their own accord. Longitudinal tracking capabilities will help quantify the benefits of time on task with CATE. The tracking will also allow us to test the above hypothesis and quantify benefits. We are also curious about the benefits of CATE for certain students, for example those who study alone.

As we move forward we want to maintain the ability for students to access CATE who may have unreliable Internet service. The current license agreement allows users download (and even modify) the source. Once downloaded, CATE may be run from a local file. No user management features will be available when running in this mode, but problems with intermittent connectivity can be mitigated.

We would like to thank and acknowledge the contributions of Wayne Pilkington towards this effort.

\section{Example of Analysis by Equivalent Circuits}

The sequence of images in Figures 6.a through 6.p illustrates the major steps in an example of analysis by equivalent circuits. At each stage in the sequence CATE presents variations of the original circuit with explanations of equivalencies and computed quantities. This method of analysis illustrates many learning objectives discussed in Section 2. 


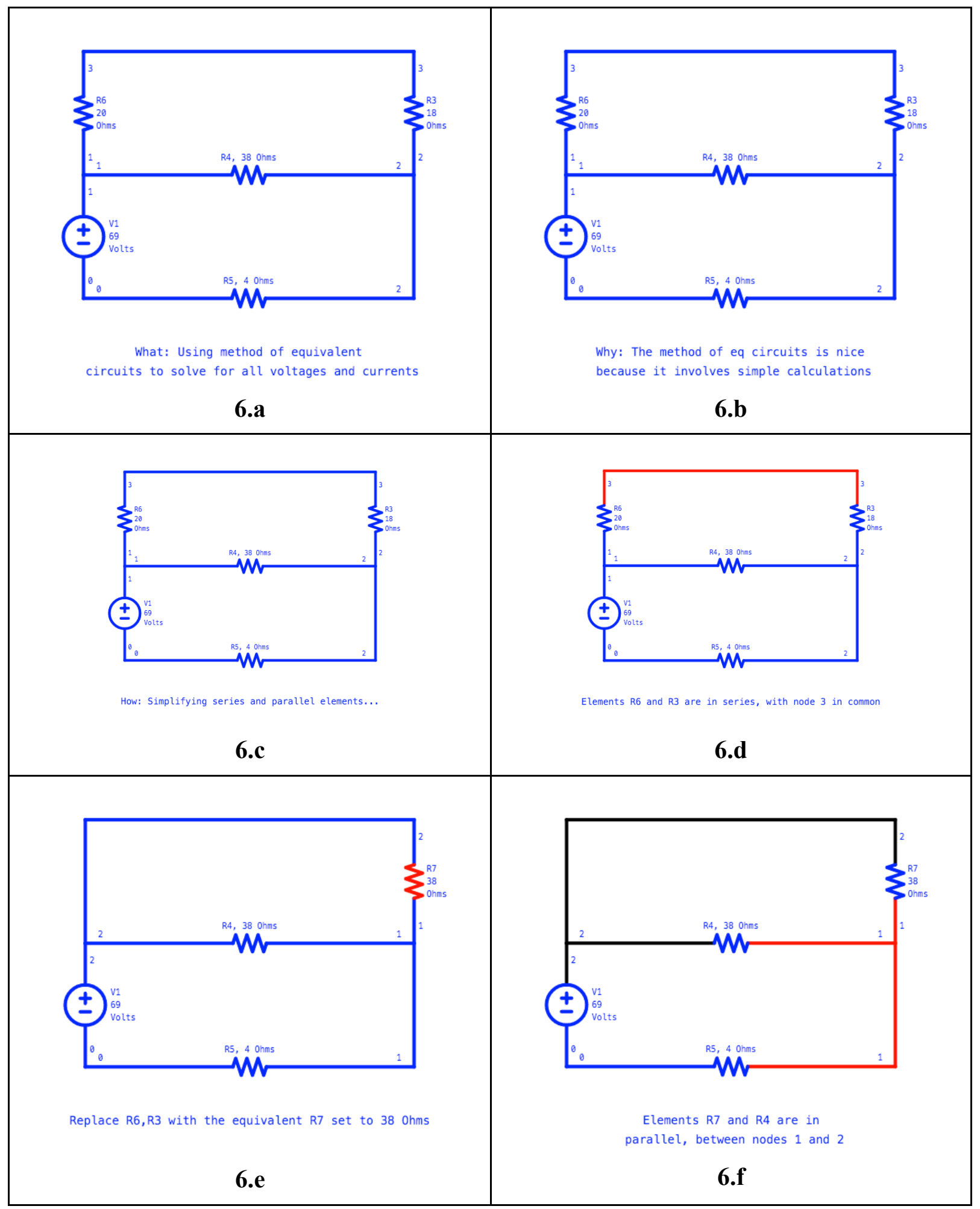




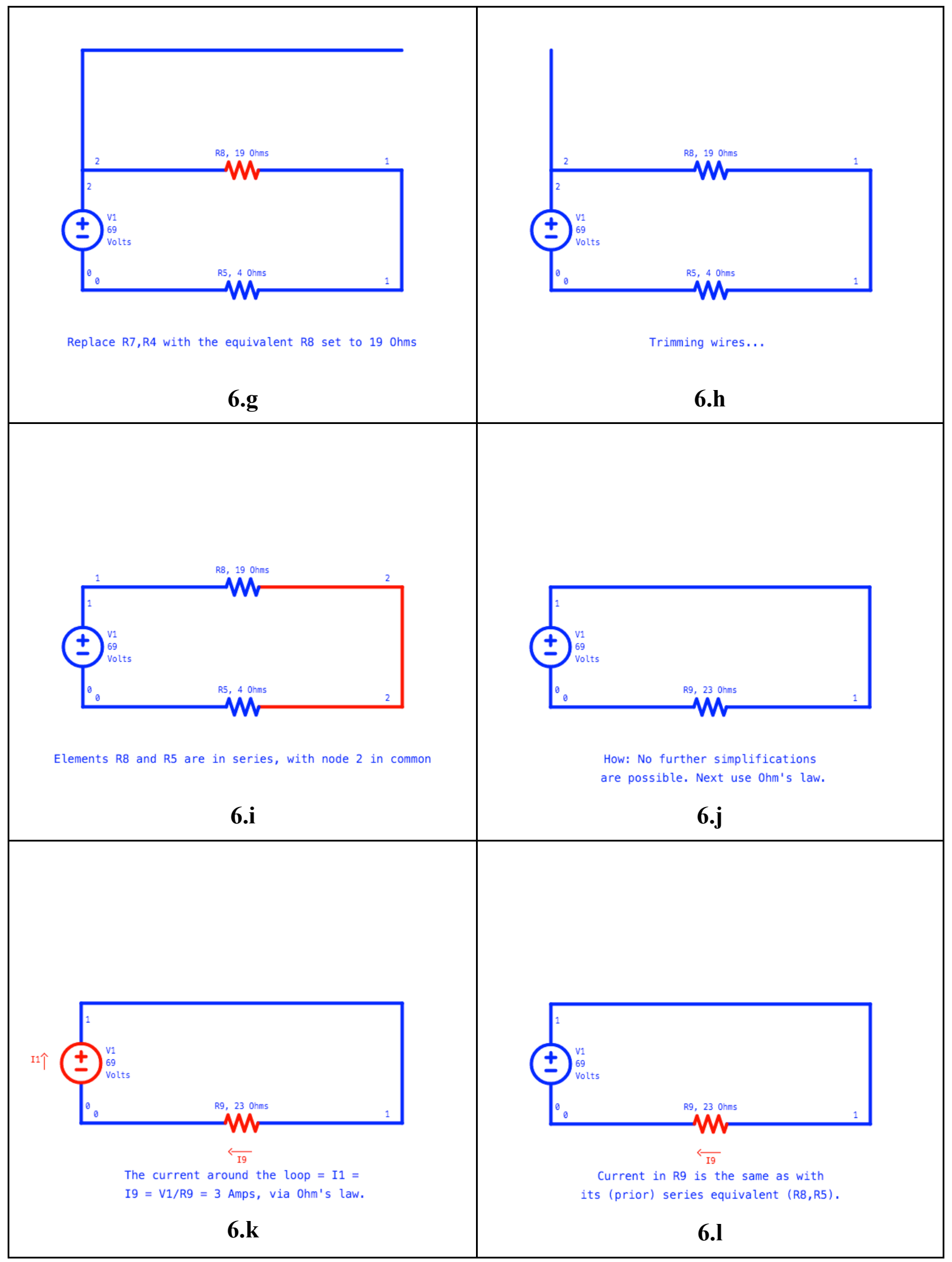




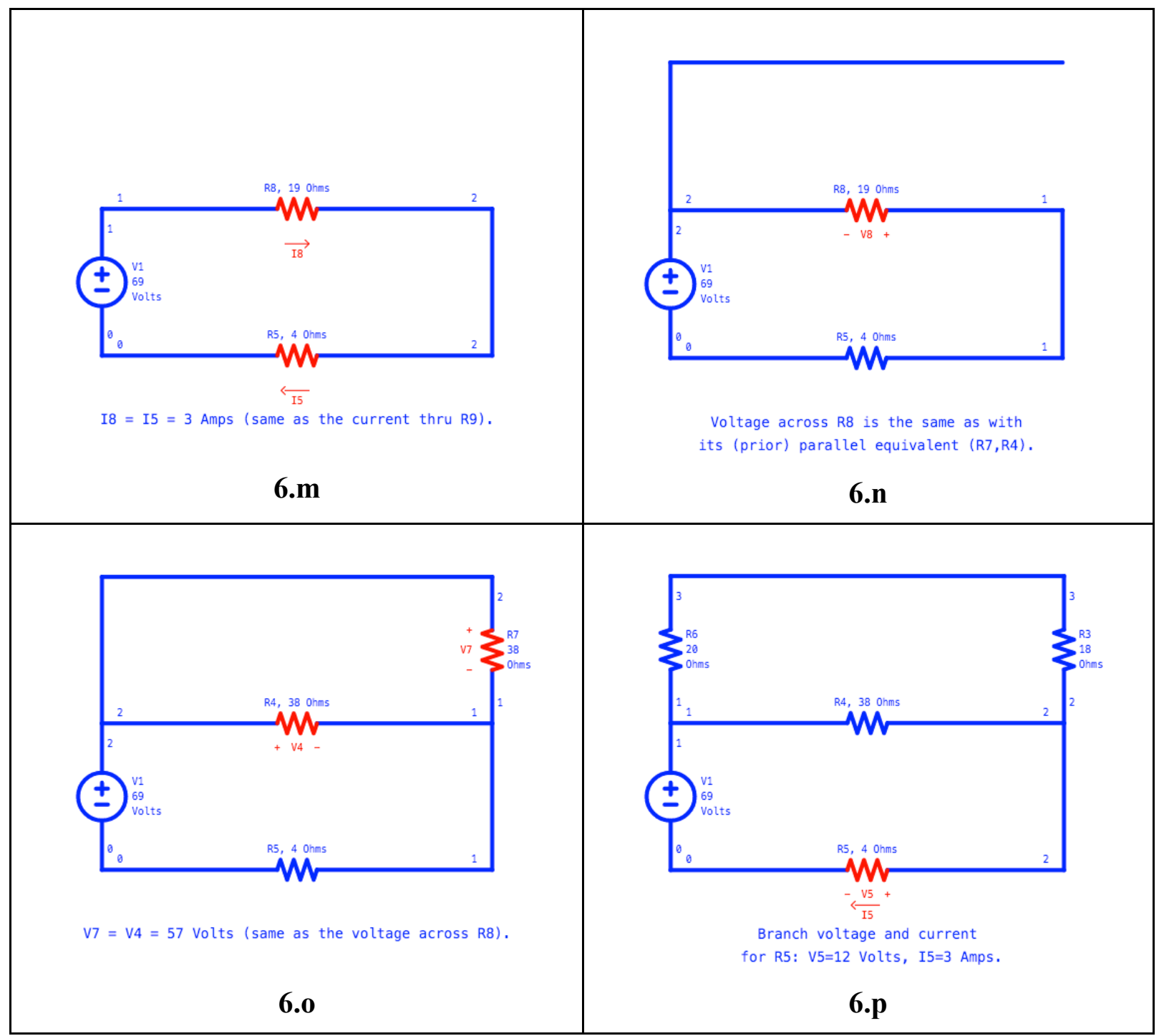

Figures 6.a to 6.p, illustrating the major steps in analysis by equivalent circuits, as presented to a student by CATE.

7. References

1. P. Minhas, A. Ghosh, L. Swanzy, "The Effects of Passive and Active Learning on Student Preference and Performance in an Undergraduate Basic Science Course," Anatomical Sciences Education, v5 n4 p200-207, Jul-Aug, 2012.

2. Abdullah, M.H., Self-directed learning. ERIC digest. Bloomington, IN: Indiana University, ERIC Clearinghouse on Reading, English and Communication, 2001.

3. Evaluation of Evidence-Based Practices in Online Learning: A Meta-Analysis and Review of Online Learning Studies, U.S. Department of Education, Office of Planning, Evaluation, and Policy Development, Washington, D.C., 2010.

4. P. V. Engelhardt and R. J. Beichner, "Students' understanding of direct current resistive electrical circuits," Am. J. Phys. 72, 98, 2004.

5. M. Nahvi and J. Edminister, "Schaum's Outline of Electric Circuits," Sixth Edition, McGraw-Hill Education, 2014. 
6. H. N. Mok, "Teaching Tip: The Flipped Classroom,” Journal of Information Systems Education, 25(1), Spring 2014.

7. O. S. Oluwatumbi, "E-Classroom of the 21st Century: Information Gaps," Journal of Education and Practice, Vol.6, No.18, 2015.

8. Waldorf, D. J., and Schlemer, L. T., "The Inside-Out Classroom: A Win-Win-Win Strategy for Teaching with Technology," Computers in Education Journal, 3 (1) 2012.

9. Harb, J. N., Durrant, S. O., and Terry, R. E., "Use of the Kolb Learning Cycle and the 4MAT System in Engineering Education" Journal of Engineering Education, 82(2), 1993.

10. tinyURL.com/CATE-INTRO

11. YourLearningCoach.com

12. F. W. DePiero, "Work-in-Progress: Design of an Online Learning Coach," in Proceedings of the 2013 ASEE Annual Conference \& Exposition. Atlanta, GA, ASEE, 2013.

13. moodle.org

14. mooc.org

15. bwrcs.eecs.berkeley.edu/Classes/IcBook/SPICE

16. C. D. Whitlatch, Q. Wang, and B. J. Skromme, “Automated problem and solution generation software for computer-aided instruction in elementary linear circuit analysis," in Proceedings of the 2012 ASEE Annual Conference \& Exposition. Washington, D.C., ASEE, 2012.

17. B. J. Skromme et al., "Teaching linear circuit analysis techniques with computers," in Proceedings of the 2013 ASEE Annual Conference \& Exposition. Atlanta, GA, ASEE, 2013.

18. B. J. Skromme et al., "Computer-aided instruction for introductory linear circuit analysis," in Proceedings of the 2015 Frontiers in Education Conference, El Paso, TX, FIE, 2015.

19. L. Weyten, P. Rombouts, and J. De Maeyer, "Web-based trainer for electrical circuit analysis," IEEE Trans. Educ. 52, 185, 2009.

20. javascript.com

21. mathjs.org

22. http://ijcai.org/Past Proceedings/IJCAI-85-VOL1/PDF/005.pdf

23. B. P. Butz, M. Duarte, and S. M. Miller, "An intelligent tutoring system for circuit analysis," IEEE Trans. Educ., vol. 49, pp. 216-223, 2006..

24. W. H. Hayt Jr., J. E. Kemmerly, and S. M. Durbin, Engineering Circuit Analysis, McGraw-Hill, New York, 2011.

25. J. W. Nilsson and S. A. Riedel, Electric Circuits, Prentice-Hall, Boston, 2011.

26. Roger Azevedo and Michael J Jacobson, Advances in scaffolding learning with hypertext and hypermedia: a summary and critical analysis, Educational Technology, Research and Development, Feb 2008, 56, 1, Research Library, p 93.

27. Skromme, B., \& Robinson, D. H. (2015). Addressing Barriers to Learning in Linear Circuit Analysis. In ASEE Annual Conference (pp. 26.158.1-26.158.15). Seattle, WA. Retrieved from https://peer.asee.org/23497 